\title{
Garantías del derecho a protección y cuidados especiales orientado a la sexualidad de adolescentes salvadoreños
}

\author{
Carolina Lucero Morán \\ Docente Utec \\ carolina.moran@utec.edu.sv \\ Recibido: 22/11/2016 - Aceptado: 24/01/2017
}

\section{Resumen}

La investigación tuvo por objetivo hacer un diagnóstico del grado de cumplimiento de las garantías del derecho a la protección y cuidados especiales de adolescentes salvadoreños, orientado a la sexualidad, y establecer las influencias que más violentan el derecho a la salud sexual y reproductiva, conforme el Art. 32 de la Ley de Protección Integral de la Niñez y Adolescencia (Lepina).

Los participantes fueron adolescentes salvadoreños con edades de entre 12 y 18 años, de los catorce departamentos del país, escogidos al azar en un muestreo simple estratificado con un nivel de confianza del $95 \%$ y un margen de error del $2 \%$. La investigación fue de tipo descriptivo, mediante muestras probabilísticas con uso de encuestas (Montero y León, 2007).

Entre los hallazgos principales se tiene que los jóvenes perciben haber recibido educación sexual y formación, mientras que las prácticas de salud sexual aparecen con medias bajas; que tanto la Iglesia como la escuela tienen una tendencia positiva a servir como referentes de educación sexual, para los estudiantes más jóvenes; que internet permite a los jóvenes un amplio acceso a

\section{Abstract}

This research focused on conducting a diagnosis of the degree of compliance of the guarantees of right for the protection and special care of Salvadorean adolescentsoriented to their sexuality-- and to establish the influential factors that mostly violate their right to sexual and reproductive health, in compliance to Art. 32 of the Child and Youth Integral Protection Act (Ley de Protección Integral de la Niñez y Adolescencia, Lepina, given its Spanish acronym).

The population included Salvadorean adolescents ages 12 through 18, from the fourteen departments of the country; they were randomly selected via a simple stratified sampling, with a confidence of $95 \%$ and a margin of error of $2 \%$. This is a descriptive research that used probabilistic sampling and surveys (Montero y León, 2007).

Amongst the most relevant findings we can mention the fact that the youth do perceive having received sexual education and training, while their sexual health practices show low medians. Both the Church and the school tend to positively serve as referents in sexual education for

Docente investigadora de la Facultad de Derecho de la Universidad Tecnológica de El Salvador (Utec). Especialista Derecho Ambiental. Coordinadora de la unidad de Investigación Jurídica. 
contenidos de tipo sexual; que el control de los padres sobre los hijos disminuye al aumentar la escolaridad de estos; y que existe una confianza mediana en la orientación sobre estos temas que los padres dan a sus hijos.

\section{Palabras clave}

Educación sexual para jóvenes-enseñanza, orientación sexual, derechos del niño, bienestar infantil-El Salvador. the youngest students; the internet allows adolescents ample access to sexual related content; parental control over kids decreases in relation to the number of years the latter ones complete at school; finally, there is a median level of confidence in the orientation parents give their children towards these topics.

\section{Keywords}

Sexual education for adolescents-teaching, sexual orientation, children's rights, child welfare-El Salvador.

respaldada por la Convención de Derechos de la Niñez (1990) y el Código de Familia (1993), declara susceptible de protección a todo menor de 18 años, es menester definir iniciativas para mermar en los adolescentes la libre disposición de aventurarse en relaciones sexuales que puedan entorpecer su desarrollo personal y social.

lo enunciado en la Declaración Universal de Derecho Humanos (1948), artículo 25, numeral 2, de la cual, más tarde, se desprende la Convención sobre los Derechos del Niño. De acuerdo con el Fondo de las Naciones Unidas para la Infancia, Unicef (2012), las niñas, niños y adolescentes precisan muy especialmente de protección y cuidados especiales.

Con frecuencia estos grupos se involucran en relaciones sexuales que tienen como consecuencia embarazos precoces (Cataño, Restrepo, Portilla y Ramírez, 2008); sus derechos humanos no se velan adecuadamente. Artículos tales como el 24, literal d), de la Convención de Derechos de la Niñez, que manda asegurar la atención sanitaria prenatal y postnatal apropiada a las madres menores de edad, el cual es retomado en la Lepina en los artículos 21, 22, 23 y 24; el Art. 2 de la misma Convención, que indica respetar los derechos sin distinción de raza, sexo, clase social, etc., y también plasmado en el Art.11 de la Lepina; o el Art. 3, numeral 2, de la Convención, que manda el compromiso de "asegurar al niño la protección y el cuidado que sean necesarios para su bienestar, teniendo en cuenta los derechos y deberes de sus padres, tutores $u$ otras personas responsables de él ante la ley, y, con ese fin, tomarán todas las medidas legislativas y administrativas adecuadas", retomado este en los artículos $12,13,14$ y 112 de la Lepina.

Siendo que en El Salvador las niñas, los niños y los adolescentes están protegidos por la Lepina (2010), la cual,
La Lepina propone no solo una salud médica y psicológica, sino una salud sexual y reproductiva, indicando en su Art. 32 que todas las niñas y todos los niños y adolescentes, según sea su desarrollo físico, psicológico y emocional, tienen el derecho a recibir información y educación en salud sexual y reproductiva, de forma prioritaria por su madre y su padre. Agrega que "el Estado garantizará la existencia y el acceso a los servicios y programas de salud y educación sexual integral para la niñez y la adolescencia, con el objeto de fortalecer su realización personal, prevenir infecciones de transmisión sexual, disminuir riesgos de abuso sexual y prepararlos para una maternidad y paternidad responsable en la adultez, sana y sin riesgos".

El Art. 3 de la Lepina (2010), en sus definición de niña, niño y adolescente, nos dice que "se presumirá niña o niño toda persona desde el instante mismo de la concepción hasta los doce años cumplidos, y adolescente es la comprendida desde los doce años cumplidos hasta que cumpla los dieciocho años de edad". La investigación ha estado regida por dicho parámetro de edad.

El tema de la sexualidad en adolescentes necesita ser abordado urgentemente. Dada la desinformación, o dado el exceso de información, que impera en nuestro país, los jóvenes acceden de hecho a las relaciones sexuales, aunque no tengan derecho. 
El asidero del derecho de los adolescentes a la protección y a los cuidados especiales se encuentra en la Declaración Universal de Derechos Humanos y en la Declaración de los Derechos de la Niñez, proclamadas por la Asamblea General de Naciones Unidas (1959), la Convención sobre los Derechos de la Niñez (1990), el Código de Familia, la Lepina y, por supuesto, la IV Conferencia Mundial sobre la Mujer y la población celebrada en Beijing.

Se ha pretendido no solo escudriñar en la defensa y aplicación del derecho a la protección y cuidados especiales, enunciado en la Declaración Universal de Derechos Humanos (1948), sino indagar un poco en ese concepto que cita Delors et al (1996): "El sector de educación juega un importante papel en la preparación de niños, niñas y jóvenes para asumir papeles y responsabilidades propias del mundo adulto".

Los objetivos de esta investigación fueron los siguientes: 1) hacer un diagnóstico del nivel de cumplimiento de las garantías del derecho a la protección y cuidados especiales de adolescentes salvadoreños, 2) determinar las actitudes más frecuentes que generan inclinación a las relaciones sexuales en menores de 18 años, 3) identificar el grado en que las instituciones relacionadas con adolescentes brindan orientación sexual, 4) establecer influencias negativas que más acuden a la violencia del derecho a la salud sexual y reproductiva, 5) indagar si los adolescentes tienen conocimiento de que existe una ley que protege sus derechos y 6) determinar usos y prácticas sexuales de los adolescentes.

Origen de los derechos de la niña, del niño y de los adolescentes y del derecho a la protección y cuidados especiales

Jules Vallès, en su obra El niño (1879), emite: "Defenderé los derechos el niño, como otros defienden los derechos del hombre". En 1892 se conoce también la reflexión sobre los derechos del niño de Kate D. Wiggin en Children's Rights (Rojas, F., 2007).

Fue en 1924 cuando surge la primera declaración oficial de derechos del niño, denominada como Declaración de Ginebra; redactada por Eglantyne Jebb, fundadora de Save the Children, y aprobada por la Sociedad de Naciones el 26 de diciembre de 1924.
En 1948 se aprueba la Declaración Universal de los Derechos Humanos. Esta deja entrever, en sus artículos 16, 25 y 26, la preocupación por la niñez; en su artículo 25 , numeral dos, acerca del derecho a cuidados y asistencia especiales de la niñez.

Posteriormente, en 1959, la Organización de las Naciones Unidas, ONU, aprueba la Declaración de los Derechos del Niño, concretando para los niños los derechos contemplados en la Declaración Universal de Derechos Humanos. En su preámbulo, señala que el niño, por su falta de madurez física y mental, necesita protección y cuidado especiales, incluso la debida protección legal, tanto antes como después del nacimiento.

A partir de 1979, con ocasión del Año Internacional del Niño, se comenzó a discutir una nueva declaración de derechos del niño, fundada en nuevos principios. En 1989 se firmó en la ONU la Convención sobre los Derechos del Niño, ratificada por la mayoría de países, que entró en vigor el 2 de septiembre de 1990. Ratificada por El Salvador, incorporó el contenido de la Convención en el Código de Familia (1994). El Libro Tercero, Título I, define sobre los derechos y deberes de los hijos, en un capítulo único, cuyo artículo 203 señala que son derechos de los hijos: " $3^{\circ}$. Recibir de sus padres: crianza, educación, protección, asistencia y seguridad". Posteriormente, el Capítulo II, en Cuidado Personal, el artículo 211, sobre la crianza, establece en su inciso primero: "El padre y la madre deberán criar a sus hijos con esmero; proporcionarles un hogar estable, alimentos adecuados y proveerlos de todo lo necesario para el desarrollo normal de su personalidad, hasta que cumplan la mayoría de edad. En la función de cuidado debe tenerse en cuenta las capacidades, aptitudes e inclinaciones del hijo".

El Art. 213 aborda la formación moral y religiosa; y el Art. 214, los deberes de educación, a efecto de formar integralmente a los hijos. Le siguen corrección y orientación, entre otros.

La Lepina surge en 2010 como exigencia de comités internacionales vigilantes de los derechos de la niñez. De este cuerpo, el artículo que corresponde a la temática es el 32, que atañe al derecho a la educación en salud sexual y reproductiva, como esencial en la protección y cuidados especiales a que tiene derecho todo menor de 18 años en El Salvador. 
De los informes de cumplimiento de derechos del niño y la niña a la Lepina

Luego de la implantación del Código de Familia, los comités monitores de derechos humanos de la Organización de las Naciones Unidas (ONU), el Consejo de Derechos Humanos de Naciones Unidas y el Comité de Derechos del niño y la niña, en sus informes de El Salvador, realizaron observaciones a la situación de la niñez en El Salvador (Cladem, 2010).

En las observaciones finales sobre el segundo informe presentado por el Estado, adoptadas el 4 de junio de 2004, la observación 24 recomienda que el Estado revise la normativa referente a la posibilidad de casarse antes de la edad mínima general de 18 años para aumentar la edad mínima, en este paso únicamente, y que sea igual para varones y niñas. Habría que realizar campañas de sensibilización que impidan el matrimonio precoz. Señalamiento basado en los altos índices de menores que hacen pareja antes de cumplir la mayoría de edad. La United Nations Educational, Scientific and Cultural Organization, (Unesco, 2010), señala que una educación en sexualidad efectiva puede entregar a las personas jóvenes información culturalmente relevante, científicamente rigurosa y apropiada a la edad del estudiante, lo que evitaría contraer nupcias por las causas generalmente conocidas.

La observación 52, "Salud de los adolescentes", recomienda que se intensifique la educación sexual y la enseñanza de la salud reproductiva a esa edad para reducir enfermedades de transmisión sexual (ETS) y embarazo en la adolescencia. Debe prestarse asimismo, asistencia a las adolescentes embarazadas y darles acceso a la atención de la salud y la educación. A este respecto, el Ministerio de Salud (Minsal) dio a conocer, en 2006, el "Manual de adolescentes. Guía metodológica para el facilitador y Cuaderno de trabajo para adolescentes de 15-19 años" (Minsal, 2006), cuyo objetivo es hacer accesible a los adolescentes información sobre habilidades para la vida, la salud sexual y reproductiva, que contribuya al ejercicio de una sexualidad responsable. Es en la Lepina (2010), Art. 32, donde se concreta por fin este derecho.

En la observación 52, Malos tratos y descuido, el Comité muestra preocupación por el alto grado de violencia contenida en masivas campañas de información a las que los adolescentes están sometidos.
En la observación 60, Salud de los adolescentes, el Comité reitera la inquietud por los embarazos de adolescentes y la falta de resultados de las medidas preventivas adoptadas por el Estado. Se preocupa también por la criminalidad del aborto, que lleva a los jóvenes a recurrir a métodos peligrosos y clandestinos, con consecuencias fatales.

En la observación 61, el Comité recomienda al Estado continuar promoviendo y asegurando el acceso a los servicios de salud reproductiva para todos los adolescentes para prevenir embarazos precoces; y revisar el Código Penal en lo relativo a la terminación del embarazo en todas las circunstancias. Así mismo, en la observación 68 expresa preocupación por la deserción escolar a causa del embarazo durante la adolescencia.

Otras observaciones incluyen tomar medidas en los casos de venta, trata y explotación sexual, los cuales exponen a una cultura de suyo deformante, orientando a los adolescentes a obtener no una salud, sino lo contrario al objetivo propuesto: una enfermedad sexual y reproductiva.

Adolescencia: rango de edad de los 12 a los 18 años, conforme a la Lepina

Adolescencia viene de "adolecer", de "carecer de algo", de "padecer"; deriva del latín adolescentia; esta es la edad que sucede a la niñez y que transcurre desde la pubertad hasta el completo desarrollo del organismo (Drae, 2012).

La Unicef (2011) establece que la adolescencia no es solamente una etapa de vulnerabilidad; también es una época llena de oportunidades, sobre todo para las niñas. Cuanta más educación reciban las niñas, más probable es que retrasen el matrimonio y la maternidad, y que como consecuencia sus hijos gocen de mejor salud y educación. Proporcionar a los jóvenes las herramientas que necesitan para mejorar sus vidas, y motivarlos a participar en las iniciativas que buscan mejores condiciones para sus comunidades, equivale a invertir en el fortalecimiento de sus sociedades. La Lepina señala que adolescencia es el rango de edad entre los 12 y los 18 años.

\section{Salud sexual y reproductiva en adolescentes y jóvenes}

La Conferencia de El Cairo, en 1994, al tiempo que introdujo por primera vez el concepto de salud reproductiva, permitió 
superar el enfoque demografista de las anteriores políticas mundiales de población, para centrarse más en la calidad de los servicios de salud sexual y reproductiva, en la prevención dirigida a adolescentes y jóvenes y en la equidad de género. Según la Organización Mundial de la Salud (OMS), una persona es sana no solamente por no padecer enfermedades, sino también por tener bienestar físico, mental y social. Ahumada y Kowalski (2006) sostienen que la salud reproductiva es un estado general de precisamente eso. A escala internacional, se ha consolidado un planteamiento integral para los programas de salud de los jóvenes, en el que se establece un nexo entre las intervenciones en salud, con especial énfasis en la sexual y reproductiva; y un abanico de posibilidades respecto de educación, formación profesional y fomento de la participación social. La atención de la salud de los jóvenes debe ir más allá de su bienestar físico (Conapo, 2010).

La Comisión de Derechos Humanos de la ONU, en 2003, en su resolución 2003/28, preámbulo y párrafo 6, dice: "La salud sexual y la reproductiva son elementos esenciales del derecho de toda persona al disfrute del más alto nivel posible de la salud física y mental" (ONU, 2003). Este derecho debe ir de la mano con aquellas políticas y estrategias de EI Salvador encaminadas a propiciar esa salud.

Una mala salud sexual y reproductiva es el factor que más contribuye a la carga de enfermedades entre personas jóvenes. Garantizar que los jóvenes disfruten una buena salud sexual y reproductiva tiene sentido, tanto desde la perspectiva social como de la económica: las enfermedades, abortos inseguros y embarazos no planificados implican una onerosa carga para el presupuesto familiar y del Estado (Unesco, 2010).

En el año 2009, el Ministerio de Salud de El Salvador, Minsal, reportó 92.243 inscripciones prenatales, de las cuales el 33,2\% eran de mujeres adolescentes. Del $30,7 \%$ institucional de partos, el 31,4 \% correspondió a adolescentes, contando con el $27,7 \%$ de muertes maternas también en adolescentes (Ekelund, 2011).

El Observatorio de Igualdad de Género de Latinoamérica y el Caribe, en su informe anual de 2011, asegura que la mayor proporción de embarazos de adolescentes en Latinoamérica ocurre en Nicaragua; casi 110 mujeres de cada mil adolescentes (entre 15 y 19) son madres. La proporción de mujeres nicaragüenses de 20 a 24 años que han tenido un hijo antes de los 15 años fue 11 veces mayor entre las mujeres con 3 años o menos de escolaridad que entre sus contrapartes con 7 o más años de educación formal (Actalianza, 2011).

Una mala salud sexual y reproductiva despierta el interés de los adolescentes en la aventura de la exploración emocional de la sexualidad, acarreándose experiencias adversas. Una de las principales consecuencias sociales de los embarazos en adolescentes y jóvenes es el abandono de los hijos al nacer, lo cual es del 5 al $9 \%$; madres solteras, un $40 \%$, además del abandono escolar, ingreso al mercado laboral informal y mantenerse en el ciclo de pobreza.

El Fondo de Población de las Naciones Unidas (UNFPA, 2012), sostiene que las probabilidades de que las adolescentes de entre 15 y 19 años de edad mueran debido a complicaciones durante el embarazo, o en el parto, son dos veces mayores que las de una mujer de 20 a 30 años.

\section{Derecho a educación en salud sexual y reproductiva}

En 1997, la Quinta Conferencia Internacional sobre Educación de Adultos, celebrada en Hamburgo, Alemania, abordó las problemáticas del analfabetismo, la baja calidad de la educación y la falta de oportunidades para las personas jóvenes y adultas en Latinoamérica y el Caribe (Cabello, 2006). La conferencia y otros organismos posteriores dieron lugar a diversos paradigmas enmarcados en la Convención de Derechos de la Niñez y a la necesidad de su estricto cumplimiento (Conapo, 2009). La conclusión fue que debían amarrarse las políticas a una educación en salud sexual y reproductiva. Cada día son menos los adolescentes que reciben una preparación adecuada para su vida sexual, haciéndose potencialmente vulnerables, ante el abuso y la explotación sexual, a las infecciones de transmisión sexual (ITS), incluyendo el VIH (Unesco, 2010).

Morlachetti (2006) sugiere analizar esas políticas desde un enfoque de derechos de los adolescentes y jóvenes, teniendo en cuenta sus derechos humanos, y en especial los derechos reproductivos, a la luz de los diversos compromisos contraídos por los países al ratificar los tratados internacionales de derechos humanos. En 2000, el Foro Mundial de Educación, celebrado en Dakar, resaltó, entre las propuestas para el año 2015, "otorgar mayor prioridad a la alfabetización y educación de jóvenes y adultos... especialmente a aquellos en situación de vulnerabilidad" (Cabello, 2006). 
El UNFPA (2012) asegura que, a escala global, muchos adolescentes son sexualmente activos antes de cumplir 20 años de edad, y la gran mayoría (60 \%) no utiliza ninguna protección contra el embarazo. A consecuencia de ello, cada año, 16 millones de adolescentes dan a luz en el mundo. El $90 \%$ de estos embarazos ocurre en países en desarrollo; $38 \%$ sucede en Latinoamérica y el Caribe. Los riesgos de un embarazo en la adolescencia están fuertemente asociados con las desigualdades, la pobreza y la inequidad de género (Cepal, 2012). La Unesco (2010) señala que la importancia de entregar a las personas jóvenes conocimientos y habilidades que les permitan hacer elecciones responsables en sus vidas es un tema de creciente preocupación en todos los países, particularmente en un contexto de alta exposición a materiales sexualmente explícitos que internet y otros medios hacen posible.

\section{Derechos sexuales y reproductivos de adolescentes}

En el año 2005, a partir del trabajo de la Organización Iberoamericana de Juventud (OIJ), 16 países aprueban la Convención Iberoamericana de Derechos de los Jóvenes. Conforme a esta, y otros pactos de derechos humanos, resultan relevantes el derecho a la identidad, el derecho a la vida libre de violencia, el derecho a la igualdad de oportunidades y emancipación, el derecho a la libre asociación y participación y el derecho a la justicia. Mesa et al (2006) establecen que, teniendo en cuenta que la expresión "derechos sexuales" es reciente en el lenguaje de la ONU, hay que considerar que por principio esta se incorpore dentro del concepto de salud reproductiva.

Ahumada y Kowalski (2006) enfatizan en los derechos humanos que protegen los derechos sexuales y reproductivos de adolescentes: el derecho a la vida, la salud, la educación e información, la privacidad; a decidir el número y espaciamiento de los hijos, a consentir al matrimonio y a su equidad, a vivir sin discriminación, a no sufrir prácticas que perjudiquen a las niñas y a vivir sin violencia.

La consideración de adolescentes y jóvenes como sujetos de derechos reproductivos es garantizada en la Conferencia Internacional sobre Población y Desarrollo realizada en El Cairo en 1994, reafirmado en las conferencias internacionales de Copenhague (Cumbre Mundial del Desarrollo Social) y en la IV Conferencia Mundial sobre la Mujer, celebrada en Beijing en 1995, y también considerado en la Cumbre del Milenio del año 2005, al sostener que la salud sexual y reproductiva es clave en la reducción de la pobreza y el logro del desarrollo humano (Morlachetti, 2006).

Definir si la práctica de la sexualidad es un derecho que se debe ejercer, un instinto que se ha de ejecutar o un mandato que se debe obedecer ha sido una preocupación a través del tiempo. No se puede negar que la maternidad, la paternidad, etc., son ejercidos desde edad temprana, a manera de apuro, lo que hace entrar al sistema en una contradicción en la que se hace necesario contemplar la licitud de unos derechos sexuales y reproductivos que actualmente no están tutelados por el Estado; y no únicamente la velación del derecho a la salud sexual que sugiere el Art. 32 de la Lepina, o como sugieren Fernández y Mazzotti (2003): "Es que el reconocimiento y ejercicio de los derechos sexuales y reproductivos de los adolescentes requieren el desarrollo de espacios de diálogo y conversación sobre los deseos, temores, expectativas e inquietudes que chicas y varones se formulan sobre sí mismos y sus pares". Sin embargo, es necesario analizar la posibilidad de estudiar, tal como sugieren Moldenhauer y Ortega (2004), la posibilidad de "incluir de manera transversal el lenguaje y la conceptualización de derechos sexuales y reproductivos en todas las sistematizaciones", puesto que hasta hoy no lo están.

Hablar de los derechos sexuales de los adolescentes, en El Salvador, es la constante preocupación de los diversos organismos, considerando que "el niño, por su falta de madurez física y mental, necesita protección y cuidados especiales, incluso la debida protección legal, tanto antes como después del nacimiento". Y es precisamente esa inmadurez la que ocasiona que el adolescente caiga en continuos desaciertos que aligeran su paso por la vida.

\section{Diseño metodológico}

Se realizó un estudio de tipo descriptivo mediante muestras probabilísticas con uso de encuestas (Montero y León, 2007).

\section{Participantes}

Los participantes de la investigación fueron jóvenes de 12 a 13 , de 14 a 16 y de 17 a 18 años de edad.

Se sondearon variables que pudiesen incidir directa 0 indirectamente en la salud sexual y reproductiva de la adolescencia, como la religión y el trabajo; $y$, en congruencia con la edad de la población, apareció que solamente el $12,1 \%$ de los participantes cuentan con un empleo. 
Un aspecto muy importante de la práctica, conocimiento y entendimiento de la salud sexual y reproductiva es el nivel educativo, que en la población aparece distribuido de forma bastante heterogénea. Aquí se tiene que el 0,1\% de los adolescentes cuentan con un nivel educativo menor a cuarto grado; $0,6 \%(n=14)$ posee escolaridad hasta el quinto grado; $5,8 \%(n=129)$ cursaba, al momento de la encuesta, el sexto grado; $14 \%(n=311)$ fue de séptimo; $16,8 \%(n=373)$ de octavo; $14,8 \%(n=330)$ de noveno; $26,7 \%(n=595)$ de primer año de bachillerato; $9,1 \%(n=$ $203)$ de segundo; $5,7 \%(n=127)$ cursaba tercero; y el $6,3 \%$ $(n=140)$ asistía a primer año de universidad.
En cuanto a la conformación del hogar, apareció que el 50,7 \% $(n=1.129)$ de la población vive con ambos padres, mientras que aquellos que viven solo con su madre representan el $24,8 \%$ ( $n=552)$, así como los que viven solo con su padre son el $4 \%(n=89)$, y los que viven con tíos o abuelos son el $18,7 \%(n=417)$. Apareció un $1,7 \%(n=38)$ que no vive con ningún miembro de su familia. Dicha conformación refleja, entre otras cosas, el paulatino cambio que los modelos familiares de El Salvador están experimentando.

Tabla 1. Características sociodemográficas de los participantes

\begin{tabular}{|c|c|c|}
\hline Característica & $\mathrm{F}$ & $\%$ \\
\hline \multicolumn{3}{|l|}{ Sexo } \\
\hline Masculino & 1.069 & 48 \\
\hline Femenino & 1.157 & 52 \\
\hline \multicolumn{3}{|l|}{ Edad } \\
\hline 12 a 13 años & 326 & 14.6 \\
\hline 14 a 16 años & 1.189 & 53.4 \\
\hline 17 a 18 años & 706 & 31.7 \\
\hline \multicolumn{3}{|l|}{ Religión } \\
\hline Católica & 1.252 & 56.2 \\
\hline Protestante & 973 & 43.7 \\
\hline \multicolumn{3}{|l|}{ Trabaja } \\
\hline Cuenta con un trabajo & 269 & 12.1 \\
\hline No cuenta con un trabajo & 1.950 & 87.6 \\
\hline \multicolumn{3}{|l|}{ Educación } \\
\hline $4^{\circ}$ grado o menos & 3 & 0.1 \\
\hline $5^{\circ}$ grado & 14 & 0.6 \\
\hline $6^{\circ}$ grado & 129 & 5.8 \\
\hline $7^{\circ}$ grado & 311 & 14 \\
\hline $8^{\circ}$ grado & 373 & 16.8 \\
\hline $9^{\circ}$ grado & 330 & 14.8 \\
\hline $1^{\text {er }}$ año & 595 & 26.7 \\
\hline $2^{\circ}$ año & 203 & 9.1 \\
\hline $3^{\text {er }}$ año & 127 & 5.7 \\
\hline Universidad & 140 & 6.3 \\
\hline
\end{tabular}

Tabla de elaboración propia. 


\section{Instrumento}

Para la validación del instrumento se realizó un análisis factorial exploratorio, cuya validez fue comprobada por medio de una prueba de Kaiser-Meyer-Olkin, que, al ser aplicada a la escala, dio como resultado que está apropiada para que un análisis de este tipo se lleve a cabo (KMO = 0.833; $X=12058.968)$. El análisis se realizó con veinticinco iteraciones por ítem, con una rotación de tipo Varimax para los componentes principales. Como resultado principal, se tiene que la escala del instrumento explica el $50,63 \%$ de la varianza total, lo cual garantiza la certeza de los datos. Se identificaron cinco factores principales, entre los que están Ios siguientes: Prácticas sexuales, Acceso a información relacionada con sexualidad, Protección de los padres, Expresión y libertad sexual y Orientación sobre sexualidad.

\section{Interpretación inferencial}

Se realizó una prueba de tipo Kolmogorov-Smirnov para determinar el tipo de prueba más adecuado para la obtención de datos inferenciales, obteniéndose un valor de $\mathrm{p}$ $=0.00$ para todos los ítems, es decir, sugiriendo la utilización de pruebas no paramétricas para la comprobación de las hipótesis.

\section{Procedimiento}

Para la realización del estudio, se hizo un muestreo estratificado para determinar la población representativa de cada departamento que se debía encuestar, realizándose la recolección de datos en días hábiles y de descanso. Se hizo el procedimiento en centros escolares resultantes de la estratificación, aplicándose los cuestionarios en el aula de clase. Como discriminación a priori de los sujetos, se preguntó la edad a los participantes antes de proceder con la aplicación de las pruebas. A continuación se explicó a los participantes el propósito del cuestionario. Para el procesamiento de la información se construyó una base de datos en el programa IBM SPSS.

\section{Resultados}

La investigación reflejó que los ítems referidos a la orientación sexual y a la formación en este tema han obtenido las medias más elevadas, mientras que aquellos que indican prácticas de salud sexual, como uso del condón y conocimiento de su correcta aplicación, obtienen valores más bajos.

El internet es uno de los medios que aparecen más consolidados como agentes que permiten acceso a contenidos de tipo sexual, muy por encima de los servicios celulares, que aparecen con un nivel más bajo al esperado. Podría afirmarse entonces que, si existe, no es percibido por la juventud.

La familia, por su parte, aparece como un ente con influencia de mediana a alta, con ítems como Confío en mis padres para orientarme sobre sexo, mostrando valores superiores a 3. Es decir, existe una confianza mediana en que los padres pueden orientar sobre estos temas a sus hijos.

\section{Diferencias entre los factores estudiados por sexo}

Se realizó una prueba de tipo $U$ de Mann-Whitney para determinar la existencia de diferencias significativas por cada una de las dimensiones del estudio, de acuerdo con su sexo biológico. Al realizarse la prueba, aparecieron diferencias estadísticamente significativas para todas las variables medidas, es decir, el sexo es determinante en la percepción y realización de prácticas sexuales $(p=0.00)$, el acceso que se tiene a la información $(p=0.00)$, el tipo de protección que se recibe de los padres $(p=0.00)$, la expresión y libertad sexual $(p=0.47)$ y la orientación sobre sexualidad que se recibe $(p=0.00)$. 
Tabla 2. Prueba de U de Mann-Whitney para Sexo

\begin{tabular}{lrrrrr}
\hline & $\begin{array}{l}\text { Prácticas } \\
\text { sexuales }\end{array}$ & $\begin{array}{c}\text { Acceso a la } \\
\text { información }\end{array}$ & $\begin{array}{c}\text { Protección de } \\
\text { los padres }\end{array}$ & $\begin{array}{c}\text { Expresión y } \\
\text { libertad sexual }\end{array}$ & $\begin{array}{c}\text { Orientación } \\
\text { sobre } \\
\text { sexualidad }\end{array}$ \\
\hline U de Mann-Whitney & 497222.500 & 480323.500 & 461946.000 & 588422.000 & 559117.500 \\
W de Wilcoxon & 1167125.500 & 1149069.500 & 1033861.000 & 1160337.000 & 1131032.500 \\
Z & -9.230 & -9.128 & -10.365 & -1.984 & -3.947 \\
$\begin{array}{l}\text { Significancia asíntota } \\
\text { (bilateral) }\end{array}$ & .000 & .000 & .000 & .047 & .000 \\
\hline
\end{tabular}

a. Variable de agrupación: Sexo. Tabla de elaboración propia.

La comparación por rangos, asociada a la prueba, demostró que entre los participantes del sexo masculino existe una disposición más abierta hacia las prácticas sexuales, y una diferencia aún más amplia en la cantidad de acceso a la información, es decir, los varones están mejor informados respecto a temáticas sexuales que las niñas. Caso contrario se da respecto a la protección de los padres, donde se observa que las mujeres reciben un mayor grado de cuido y control, tanto del padre como de la madre, que los varones.

Para la expresión y libertad sexual, y orientación sobre sexualidad, las mujeres también aparecen con mayores puntajes; es decir, tienen su sexualidad más afianzada y están más abiertas sobre las diferentes orientaciones sexuales que los varones (tabla 2).

\section{Diferencias entre los factores estudiados por nivel educativo}

Una situación similar se da frente al nivel educativo. La prueba de significancia de tipo Kruskal-Wallis arrojó diferencias significativas para todos los niveles, indicando una relación directamente proporcional al reforzamiento de los factores con el aumento de nivel académico. Al revisar los rangos, se evidencia que todos los factores, a excepción de Control de los padres, son directamente proporcionales al aumento de grado, mientras que el último experimenta una disminución progresiva con cada año. Es decir, mientras mayor el grado académico, existe menor control de los padres sobre el cuidado del hijo acerca de temáticas sexuales, pudiendo deberse esto a la edad o a la confianza en el sistema educativo para resolver este tipo de temáticas; sin embargo, el desinterés es palpable. Por otro lado, los que evidencian mayor control de los padres están ubicados entre $5^{\circ}$ y $7^{\circ}$ grado, dándose el quiebre en octavo grado.

Tabla 3. Prueba Kruskal Wallis por factor para Nivel académico Educación

\begin{tabular}{lrrrrr}
\hline & $\begin{array}{c}\text { Prácticas } \\
\text { sexuales }\end{array}$ & $\begin{array}{c}\text { Acceso a la } \\
\text { información }\end{array}$ & $\begin{array}{c}\text { Protección de } \\
\text { los padres }\end{array}$ & $\begin{array}{c}\text { Expresión y } \\
\text { libertad sexual }\end{array}$ & $\begin{array}{c}\text { Orientación } \\
\text { sobre sexualidad }\end{array}$ \\
\hline Chi-cuadrado & 186.930 & 81.906 & 77.640 & 177.629 & 148.882 \\
Gl & 9 & 9 & 9 & 9 & 9 \\
Significancia asíntota & .000 & .000 & .000 & .000 & .000 \\
\hline
\end{tabular}

\footnotetext{
a. Prueba de Kruskal-Wallis.

b. Variable de agrupación: Educación.

Tabla de elaboración propia
} 


\section{Diferencias entre los factores estudiados por edad}

De los resultados obtenidos de la variable Escolaridad, fue necesario hacer una validación por medio de la edad de los participantes. El resultado de la prueba de $U$ de Mann-
Whitney aplicada a la edad demostró que, efectivamente, existen también diferencias significativas entre los distintos grupos de edad, mostrando una tendencia muy similar a la proporcionalidad registrada por los grados de educación (ver tabla 4).

Tabla 4. Prueba Kruskall Wallis para los factores según edad

\begin{tabular}{lrrrrr}
\hline & $\begin{array}{l}\text { Prácticas } \\
\text { sexuales }\end{array}$ & $\begin{array}{c}\text { Acceso a la } \\
\text { información }\end{array}$ & $\begin{array}{c}\text { Protección de } \\
\text { los padres }\end{array}$ & $\begin{array}{c}\text { Expresión y } \\
\text { libertad sexual }\end{array}$ & $\begin{array}{c}\text { Orientación } \\
\text { sobre sexualidad }\end{array}$ \\
\hline Chi-cuadrado & 114.631 & 54.540 & 60.406 & 120.444 & 42.075 \\
Gl & 2 & 2 & 2 & 2 & 2 \\
Significancia asíntota & .000 & .000 & .000 & .000 & .000 \\
\hline
\end{tabular}

a. Prueba de Kruskal-Wallis.

b. Variable de agrupación: Edad.

Tabla de elaboración propia

La distribución de los datos sugiere que, a mayor edad, aumentan todos los factores medidos en la población, excepto por Protección de los padres, la cual disminuye según se pasa de un grupo a otro. Los de mayor edad, es decir, el grupo de 17 a 18 años, tienen los valores más elevados, lo cual es congruente con la madurez física y mental que se debería tener a dicha edad.

Entre las variables sondeadas, se midió también si la religión incidía en la aparición de alguno de los factores medidos. Entre los hallazgos, tal y como se esperaba, no aparecieron diferencias significativas para ninguno de ellos, excepto para Acceso a la información (ver tabla 5).

Tabla 5. Prueba de U de Mann-Whitney según factores para Religión

\begin{tabular}{|c|c|c|c|c|c|}
\hline & $\begin{array}{l}\text { Prácticas } \\
\text { sexuales }\end{array}$ & $\begin{array}{l}\text { Acceso a la } \\
\text { información }\end{array}$ & $\begin{array}{c}\text { Protección de los } \\
\text { padres }\end{array}$ & $\begin{array}{c}\text { Expresión y } \\
\text { libertad sexual }\end{array}$ & $\begin{array}{c}\text { Orientación sobre } \\
\text { sexualidad }\end{array}$ \\
\hline U de Mann-Whitney & 592479.500 & 558949.500 & 602566.500 & 597831.500 & 584782.500 \\
\hline W de Wilcoxon & 1376857.500 & 1342075.500 & 1076417.500 & 1071682.500 & 1058633.500 \\
\hline Z & -1.275 & -3.321 & -.436 & -.751 & -1.631 \\
\hline $\begin{array}{l}\text { Significancia asíntota } \\
\text { (bilateral) }\end{array}$ & .202 & .001 & .663 & .453 & .103 \\
\hline
\end{tabular}

a. Variable de agrupación: Religión.

Tabla de elaboración propia. 
De la comparación de los rangos, de acuerdo con la dimensión, se encontró que los protestantes tienen un mayor acceso social a la información con contenido sexual que los católicos. Esto engloba no solo la exposición a contenidos sexuales nocivos, sino también a formación, lo que podría deberse a cánones y políticas que cada Iglesia maneja sobre dichas temáticas, donde en la católica existe control sobre acceso al contenido, mientras que, posiblemente, dependiendo de la Iglesia protestante que sea, no haya tanto control sobre la exposición de la juventud a dichos contenidos.

Finalmente, se sondeó si la conformación del hogar, es decir, con quién viven los jóvenes, tiene influencia en la aparición de alguno de los factores. El análisis demostró que la única instancia en la cual esto tiene influencia es en las prácticas sexuales, donde se advierte que los jóvenes que viven solo con el padre o con otro pariente muestran valores mucho más elevados que los que viven solo con la madre, o que los que viven con ambos. (tabla 6).

Tabla 6. Prueba Kruskall Wallis para Tipo de hogar

\begin{tabular}{lrrrrr}
\hline & $\begin{array}{c}\text { Prácticas } \\
\text { sexuales }\end{array}$ & $\begin{array}{c}\text { Acceso a la } \\
\text { información }\end{array}$ & $\begin{array}{c}\text { Protección de } \\
\text { los padres }\end{array}$ & $\begin{array}{c}\text { Expresión y } \\
\text { libertad sexual }\end{array}$ & $\begin{array}{c}\text { Orientación } \\
\text { sobre } \\
\text { sexualidad }\end{array}$ \\
\hline Chi-cuadrado & 11.698 & 7.535 & 6.408 & 7.460 & 5.816 \\
Gl & 4 & 4 & 4 & 4 & 4 \\
Significancia asíntota & .020 & .110 & .171 & .113 & .213 \\
\hline a. Prueba de Kruskal-Wallis. & & & & & \\
b. Variable de agrupación: Tipo de hogar. & & & & & \\
Fuente: Elaboración propia & & & & &
\end{tabular}

Los diferentes análisis conducidos evidencian que existe una situación de vulnerabilidad por edad, educación, conformación de la familia, sexo y nivel educativo de la niñez y la adolescencia sobre diversas circunstancias.

\section{Discusión}

El Art. 3 de la Convención de Derechos de la Niñez comparte significado con el Art. 12 de la Lepina, al leerse: "En la interpretación, aplicación e integración de toda norma [...] es de obligatorio cumplimiento el principio del interés superior de las niñas, niños y adolescentes, en lo relativo a asegurar su desarrollo integral y el disfrute de sus derechos y garantías. Se entiende por interés superior del niño, niña y adolescente toda situación que favorezca su desarrollo físico, espiritual, psicológico, moral y social para lograr el pleno y armonioso desenvolvimiento de su personalidad. La madre o padre tienen obligaciones comunes en lo que respecta a la crianza y desarrollo de la niña, niño o adolescente [...]". Al respecto, Cillero (1988) define el interés superior del niño como la satisfacción integral de sus derechos, y, por tanto, es una garantía, ya que toda decisión que concierna al niño y a la niña debe considerar primordialmente sus derechos.

El Art. 350 del Código de Familia aborda el interés superior, cuando dice: "Se entiende interés superior del menor todo aquello que favorezca su desarrollo físico, psicológico, moral y social para lograr el pleno y armonioso desenvolvimiento de su personalidad, y con base en ese interés, el menor tendrá prioridad para recibir atención y socorro en toda circunstancia".

La esencia del interés superior del menor está íntimamente ligada a lo dispuesto en el Art. 25, numeral 2, del derecho a la protección y cuidados especiales que nos manda la protección de la niña, del niño y adolescente en situación de riesgo.

En un alto porcentaje, los adolescentes no conocen la Lepina; y otro porcentaje cree que atiende los derechos de las mujeres. Esto se debe a la poca divulgación de derechos que existe en los medios de comunicación y a la poca 
participación del sistema educativo, pese a lo dispuesto en los artículos 87 y 95 de dicha ley. Asimismo, están expuestos tanto a contenidos sexuales nocivos como a formación. Se señala que podría deberse a los cánones y a las políticas que cada Iglesia maneja sobre dichas temáticas.

En relación con la mejor influencia de orientación sexual, tanto la Iglesia como la escuela tienen una tendencia positiva a servir como referentes para los estudiantes en temas sexuales, obteniendo valores eminentemente positivos respecto a ser referentes de educación en salud sexual entre los jóvenes, en oposición al grupo de amigos, el cual, contrario a lo esperado, muestra valores medianos. Aquí debe resguardarse la obligación del principio de corresponsabilidad, señalado en el Art.13 Lepina.

El Art. 96 Lepina establece protección frente a información nociva o inadecuada; el 97 obliga a los medios de comunicación, tales como la televisión, radio y prensa escrita, a destinar espacios para la difusión de los derechos y cuidar los programas, atendiendo a sus necesidades informativas. Las masivas campañas de información a las que los adolescentes están expuestos son, sin duda, maltrato, por cuanto dañan su salud sexual y reproductiva, deformando a través de contenidos no adecuados la sana percepción de esta dimensión de su personalidad.

Los resultados nos ofrecen el panorama de que los jóvenes están recibiendo suficiente orientación sexual y formación en este tema; sin embargo, las prácticas de salud sexual, como el uso del condón y el conocimiento de su correcta aplicación, dicen lo contrario. Los altos índices de menores que hacen pareja antes de cumplir la mayoría de edad engrosan las estadísticas. Es necesaria una educación en sexualidad efectiva que entregue a las personas jóvenes información culturalmente relevante, científicamente rigurosa y apropiada a la edad del estudiante (Unesco, 2010).

Para la variable Trabaja, y en congruencia con la edad de la población, apareció que solamente el $12,1 \%$ de los participantes (269) cuentan con un empleo, mientras que el $87,6 \%$ (1.950) no. Acerca de si realizaban actividades laborales de otro tipo no se indagó, por considerar que no era relevante en esta investigación.

Si bien, en El Salvador, debe mantenerse el llamado a educar a los adolescentes en salud sexual y reproductiva, esto no significa que se les deba permitir que asuman libremente su sexualidad. No se trata de desvirtuar la adolescencia, sino de construirla. En esto radica su derecho a la protección y cuidados especiales, en que se esté vigilante para que coronen una vida de éxito.

\section{Conclusiones}

La mayoría de adolescentes no conoce a quién está dirigida la Lepina y que esta vigila sus derechos. La mitad de la población desconoce el significado del acrónimo Lepina.

La familia aparece como un ente con influencia de mediana a alta. Existe una confianza mediana en que los padres pueden orientar sobre estos temas a sus hijos. Esto depende del nivel de acercamiento o indiferencia a la que estén expuestos los adolescentes. En los casos en que los padres trabajan o están alejados no se puede presumir buena comunicación, por lo tanto, la confianza no existe. Si el grupo familiar se mantiene unido, podría lograr que la educación sexual de los hijos sea totalmente sana. Los adolescentes indican que la comunicación entre padres e hijos es cada vez más escasa. Se considera a las ONG como una alternativa orientadora, a falta del grupo familiar.

Los adolescentes no esperan que la escuela los oriente en forma suficiente. Muestran confianza en la Iglesia y en la Biblia. Muestran confianza también en el grupo de amigos, lo que muchas veces subvierte la información recibida. Los grados de confianza aparecen repartidos respecto del hogar, la escuela, la Iglesia, grupo de amigos y medios de información comercial. El hogar y los medios de comunicación también revelan elevados índices de confianza.

El internet es un medio de información accesible a todas las esferas. Los adolescentes basan mucho de su aprendizaje en esta herramienta tecnológica; reciben mensajes que les ofrecen desde tips para conquistar a la pareja hasta modos de llevar a cabo la relación sexual. La mayoría de 'mensajitos' tienen material sexual. Los que más denuncian la situación son los del rango de 14 a 16 años de edad. El internet se consolida como agente de acceso a contenidos de tipo sexual. Los servicios mediante teléfonos celulares aparecen con un nivel más bajo. La intromisión de esta tecnología en sus vidas no es percibida por la juventud.

No existe control por parte de los organismos correspondientes sobre la información a la que el adolescente 
accede. Al menos, el 50 \% de la población considera la página de Facebook como herramienta para conocer personas, encontrar pareja y expresar su sexualidad. Las revistas sobre contenido sexual en librerías y supermercados están al alcance de la mano, y los adolescentes pueden hojearlas sin tener que comprarlas.

Hay una marcada inclinación a buscar parejas para noviazgo, sexuales, de convivientes, etc. Esto constituye un riesgo, puesto que no están adecuadamente informados al respecto. Llama mucho la atención que, en el rango de edad de 12 a 13 años, se obtuvo un total de 96 casos afirmativos.

Las frecuencias con que los adolescentes han tenido relaciones sexuales son elevadas, desde la perspectiva de la muestra de 2.226 personas. Es decir, un total de 526 señala que ha tenido relaciones con poca o mayor constancia. 5 adolescentes entre los 12 a 13 años manifestaron estar acompañados; 31 entre los 14 a 16 años; y 31 entre los 17 a 18 años. No se preguntó si la pareja mantiene sus estudios, o si este gasto aún corre por cuenta de sus padres.

Los adolescentes están teniendo relaciones sexuales desde los 12 a los 13 años. 17 personas manifiestan haber tenido relaciones sexuales con más de una pareja. En las edades de 14 a 16 años se evidencian 169. En el rango de 17 a 18 años se encontraron 161 adolescentes que también están teniendo relaciones sexuales. Aparece que el grupo de amigos tiene influencia en ello.

Las edades de 12 a 13 años revelan apenas 40 personas informadas acerca del uso del preservativo. En el rango de 14 a 16 años, encontramos 362 personas informadas; de 17 a 18 años se revelan 295. Puede notarse que las personas de 14 a 16 años de edad están mejor informadas que el resto. Esto puede ser una debilidad, si tomamos en cuenta que el total de personas que tiene más relaciones sexuales se encuentra en este grupo de edad.

Los datos obtenidos por el no uso de preservativo arrojan la preocupación de la exposición de los adolescentes a enfermedades de transmisión sexual. El grupo de 12 a 13 años de edad no usa preservativo. Inicialmente se infirió que era porque no tienen relaciones sexuales; pero sorprendentemente se encontró un grupo de 34 que admite su uso. En el grupo de 14 a 16 años, también dijeron que no lo usan 905. Este rango permitió descubrir un grupo de 283 que admite el uso del preservativo. El grupo de 17 a 18 años se encontraron 459 que dijeron que no lo usaba; y un grupo escaso de 248 dijo que sí.

1.743 manifiestan que no hacen uso de la unidad de Salud. Esto podría significar dos cosas: o no hacen uso del preservativo o lo adquieren en otro establecimiento. El rango más alto se percibe en las edades de 14 a 16 y 17 a 18 años.

Se advierte que el adolescente está solicitando que la familia ponga límites a su libertad; 1.557 personas contestaron estar de acuerdo con que sus padres franqueen sus ímpetus de sostener relaciones sexuales; mientras que 669 no. Así mismo, confían en que sus padres son los indicados para orientarlos sobre estas temáticas.

Se considera que el material de la escuela es útil e ilustrativo, pero debe mejorarse.

Llama la atención que, de la muestra, 1.612 adolescentes consideran que no es necesaria la mayoría de edad para tener relaciones sexuales; mientras que 614 opinan que sí.

La adolescencia salvadoreña no está siendo adecuadamente informada, según el Art. 32 Lepina, acerca de la salud sexual o reproductiva. Es decir, la garantía no se cumple.

En la búsqueda de la sustentación de sus derechos, se descubre que estos son violentados sistemáticamente por los amigos, los medios de comunicación, las tecnologías de información, la escuela y principalmente el grupo familiar.

Existen influencias negativas que acuden a la violencia del derecho a la salud sexual y reproductiva, tales como la falta de atención en el hogar; la falta de vigilancia en los centros educativos y la Iglesia, y la falta de programas por parte del Estado.

\section{Recomendaciones}

Se recomienda que las compañías de telefonía tengan el registro de si el aparato será usado por un menor, a efecto de restringir el acceso a los mensajitos de dudosa educación. La familia debe tener presente esta información y avisarla a la compañía telefónica. El acceso a internet debe ser vigilado por los padres, siendo este uno de los medios que aparecen más consolidados como agentes que permiten acceso a contenidos de tipo sexual. 
Efectuar, a través del instituto o centro escolar, una mayor divulgación de la Lepina, a efecto de lograr más conciencia por los adolescentes.

Fomentar en la familia un mayor nivel de confianza, para que no se pierda la posibilidad de orientar al adolescente en estos temas. La Iglesia, las ONG, Ios grupos sociales interesados, Ciudad Mujer, etc., pueden coadyuvar al rescate de la confianza de la familia salvadoreña. La familia debe poner más atención. Si los padres están muy ocupados, deben designar a una persona de confianza para que sea esta la que vigile la educación en salud sexual de sus hijos e hijas.

Deben reforzarse, en las políticas estatales, los programas de valores.

Los medios de comunicación deben reforzar, paralelamente, la publicidad y los contenidos dirigidos a la población adulta, y hacer campañas de concientización para las niñas, los niños y adolescentes.

Igualmente, el Estado debe establecer permanentemente una campaña de salud sexual y reproductiva para los adolescentes y jóvenes, a efecto de disminuir la tasa de relaciones sexuales de estos, así como restar a la cada vez más elevada tendencia a acompañarse, buscar pareja, embarazarse y contraer nupcias a temprana edad.

Las librerías y los supermercados deben mantener fuera del alcance de esta población las revistas sobre contenido sexual, o tener disposiciones de no hojear la información.

Los padres, tutores o responsables están obligados a vigilar los noviazgos de sus hijos. A este respecto, deben apegarse a lo dispuesto en el Art. 32 de la Lepina.

\section{Referencias}

Actalianza (2011). "Infome sobre derechos humanos y conflictividad en Centroamérica 2010-2011". Nicaragua: Federación Luterana Mundial.

Ahumada, C. \& Kowalski, S. (2006). Derechos sexuales y derechos reproductivos. Guía para activistas jóvenes. Canada, Ontario: The youth coalition.

Cabello, M. (2006). Educación de personas jóvenes y adultas en Iberoamérica. Madrid, España: Ministerio de Educación y Ciencia. Universidad Complutense de Madrid.

Cataño, D.; Restrepo, S.; Portilla, N. y Ramírez, H. (2008). "Autoestima y sexualidad en adolescentes: validación de una escala". Investigaciones Andinas No. 16, 108.

Cepal (2012). "Conferencia estadística de las Américas". Quito, Ecuador: Comisión Económica para América Latina y el Caribe.

Cillero, M. (1988), Infancia, ley y democracia. Santiago de Chile: Sociedad de Ediciones Universidad Diego Portales.

Cladem (2010). "Jurisprudencia sobre derechos humanos de las mujeres de El Salvador. Comités monitores de Derechos Humanos de Naciones Unidas-Consejo de Derechos Humanos de las Naciones Unidas. Lima, Perú: Susana Chiariotti. Cladem.

Código de Familia (1993). San Salvador: Asamblea Legislativa. Conapo (2010). Diagnóstico mundial de la juventud. México: Consejo Nacional de Población.

Conapo (2009). "Informe de ejecución del programa de acción de la Conferencia Inernacional sobre la población y el desarrollo 1994-2009". México: Consejo Nacional de Población.

Digestyc (2007). Dirección General de Estadísticas y Censos. Recuperado el 13 de octubre de 2012 de http://www.censos.gob.sv/util/datos/Resultados\%20 VI\%20Censo\%20de\%20Población\%20V\%20de\%20 Vivienda\%202007.pdf

Drae (2012). Diccionario de la Real Academia Española, 22. a. Edición. España: Real Academia Española.

Drae (2012). Diccionario de la Real Academia Española. Recuperado el 12 de octubre de 2012 de http://lema. rae.es/drae/?val=adolescer

Ekelund, E. (2011). "Informe sobre derechos humanos y conflictividad en Centroamérica 2010-2011". Honduras, El Salvador, Costa Rica, Guatemala: Federación Luterana Mundial.

Fespad (2002). Convención sobre los derechos del niño y protocolos facultativos explicados. San Salvador: Fespad ediciones. Fundación de Estudios para la Aplicación del Derecho.

Lepina (2010). "Ley de protección a la niñez y a la adolescencia". San Salvador, El Salvador: Gobierno de El Salvador.

Mesa, A.; Suárez, C.; Brenes, V.; Rodríguez, G.; Mayén, B. y Santos, E. (2006). "Marco internacional y nacional de los derechos sexuales de adolescentes". México: Afluentes, S.C. 
Minsal (2006). “Manual de adolescentes. Guía metodológica para el facilitador y Cuaderno de trabajo para adolescentes de 15-19 años". El Salvador: Ministerio de Salud.

Moldenhauer, N. y Ortega, M. (2004). Adolescencia y sexualidad. Chile: Universidad Austral de Chile.

Montero, I. y Ortega, M. (2007). Métodos y Técnicas de Investigación. Madrid. McGraw-Hill.

Morlachetti, A. (2006). "Políticas de salud sexual y reproductiva para adolescentes y jóvenes: Un enfoque desde los derechos humanos". Santiago de Chile: Cepal.

OIJ (2012). El Salvador y la Convención Iberoamericana. Madrid: Secretaría General de la Organización Iberoamericana de la Juventud.

OMS (2006). "Defining sexual health Report of a technical consultation on sexual health 28-31". Ginebra, Suiza: Organización Mundial de la Salud .

OMS (2002). "Sexual Health: Report of a technical consultation on sexual health". Ginebra, Suiza: Organización Mundial de la Salud.

ONU (2005). "Decenio de las Naciones Unidas para la alfabetización: la educación para todos 2003-2012". España: Facultad de Traducción de la Universidad de Salamanca.

ONU (1995). "Informe de la Cuarta Conferencia Mundial sobre la Mujer". Nueva York: Naciones Unidas ISSN 921-330155-3.

ONU (1996). "Programa de acción mundial para los jóvenes hasta el año 2000 y años subsiguientes". New York: Organización de las Naciones Unidas.
ONU (2003). "Resolución Comisión de Derechos Humanos: El Derecho al disfrute del más alto nivel posible de salud física y mental". Nueva York: Organización de las Naciones Unidas.

OPS (2000). "Perfil de la salud sexual y reproductiva de los y las adolescentes y jóvenes de América Latina y el Caribe: revisión bibliográfica, 1988-1998". Washington, USA: Organización Panamericana de la Salud.

Rojas, F. (2007). Los derechos del niño en Chile: Una aproximación histórica 1910-1930. Chile: Instituto de Historia, Pontificia Universidad Católica de Chile.

Save the children (2012). Rompamos las cadenas de la esclavitud infantil. España: Save the Children España.

Unesco (2010). Adolescencia. México: Unesco.

Unesco (2010). "Orientaciones técnicas internacionales sobre educación en sexualidad". Francia: Unicef.

Unfpa (12 de mayo de 2012). "Salud sexual y reproductiva en adolescentes y jóvenes". Recuperado el 13 de octubre de 2012 de UNFPA, México, http://www.unfpa.org.mx/ ssr_adolescentes.php

Unicef (3 de enero de 2012). "Unicef". Recuperado el 12 de abril de 2012 de http://www.unicef.org/spanish/crc/ index_using.html

Unicef (2011), Estado mundial de la infancia. New York: División de Comunicaciones, Unicef.

Vallès, J. (2012), El niño. España: ACVF Editorial, ISBN: 9788494022111.

WHO (12 de abril de 2012). "World Health Organization". Recuperado el 14 de octubre de 2012 de http://www. who.int/reproductivehealth/en/ 